\title{
A New Family of Omnidirectional and Holonomic Wheeled Platforms for Mobile Robots
}

\author{
François G. Pin, Member, IEEE, and Stephen M. Killough, Member, IEEE
}

\begin{abstract}
This paper presents the concepts for a new family of holonomic wheeled platforms that feature full omnidirectionality with simultaneous and independently controlled rotational and translational motion capabilities. We first present the "orthogonal-wheels" concept and the two major wheel assemblies on which these platforms are based. We then describe how a combination of these assemblies with appropriate control can be used to generate an omnidirectional capability for mobile robot platforms. Several alternative designs are considered, and their respective characteristics with respect to rotational and translational motion control are discussed. The design and control of a prototype platform developed to test and demonstrate the proposed concepts is then described, and experimental results illustrating the full omnidirectionality of the platform with decoupled rotational and translational degrees of freedom are presented.
\end{abstract}

\section{INTRODUCTION}

A LARGE NUMBER of wheeled or tracked platform mechanisms have been studied and developed to provide their mobility capability to teleoperated and/or autonomous robot vehicles [1]. For large and heavy outdoor robots, fourwheel, car-like driving mechanisms or skid-steer platforms have traditionally been used. Because the non-holonomic constraints ${ }^{\prime}$ on their wheel mechanisms prevents sideways movements (also termed "crab motion") without preliminary maneuvering, these vehicles are quite restricted in their motion [2]-[4], particularly when operating in tight environments. Better motion capabilities have been investigated in a variety of research centers and have been demonstrated on laboratory robots. These improvements in motion capabilities typically are derived from the use of two independent driving wheels supplemented by casters (e.g., see robot in [5]-[7]), two steerable and independently driving wheels [8], [9], or three steerable and coordinated driving wheels (e.g., see robots in [10]-[12]). The former type allows rotation of the platform around any point but does not allow sideways motion, while

Manuscript received September 9, 1992; revised January 28, 1994. This work was supported by the Office of Engineering Research Program. Basic Energy Science, of the U.S. Department of Energy, under Contract DE-AC05840R21400, with Martin Marietta Energy Systems. Inc. A patent has been applied for for some of the systems described in this paper.

F. G. Pin is with the Autonomous Robotic Systems Group, Robotics and Process Systems Div. Oak Ridge National Laboratory, Oak Ridge, TN 37831 6305 USA.

S. M. Killough is with the Robotics and Process Systems Div., Oak Ridge National Laboratory, Oak Ridge, TN 37831-6304 USA.

IEEE Log Number 9402091

'A non-holonomic constraint is a non-integrable constraint of the form $G(\bar{q}, \bar{q})=0$, binding configuration variables and their derivatives. Because the constraint is not integrable, it does not affect the space of achievable configurations, $\bar{q}$, of the system, but it restricts the space of achievable velocities $\bar{i}$ at given configurations. the second and third types realize both rotation of the platform and sideways motion through coordinated steering of the wheels. In these latter systems, however, the controls of the translational and rotational motions are not fully decoupled or independent, in the sense that very stringent compatibility conditions exist between the steering and driving velocities of the wheels [13]. To achieve the full three degrees of freedom of planar rigid body motion, these platforms must therefore be controlled as strongly constrained systems (e.g., see [14]), and any slight error in their control or actuation subsystems that violates the constraint will result in wheel slippage and accumulation of positioning errors. In addition, steering requires rotation of the wheels around a vertical axis that, for heavy payloads or vehicles equipped with wide tires, may generate significant sliding and friction of the wheels

A variety of mechanisms, inspired from the "universal wheel concept" (e.g., see [1], [15]), have been used to remedy some of the steerable wheel's friction and inter-wheel constraint problems in designing omnidirectional vehicles. A "universal wheel" is an assembly which provides a combination of constrained and unconstrained motions when turning. When two or more of these wheels are mounted on a platform, their constrained and unconstrained motions can be combined to provide omnidirectionality. The most common type of universal wheels, shown in Fig. 1 and discussed in detail in [1], involves a large wheel with many small rollers mounted on its rim. As the drive shaft turns, the wheel is driven in a normal fashion in a direction perpendicular to the axis of the drive shaft, i.e., in the constrained direction of motion. At the same time, the small rollers allow the wheel to freely move parallel to the drive shaft, providing the unconstrained direction of motion. Wheels of this type must be relatively large to accommodate the rollers and greatly suffer from the successive shocks caused when individual rollers make contact with the ground. A variation of this universal wheel incorporates elongated rollers which are positioned at $45^{\circ}$ from the axis of the main shaft of the wheel, and are tapered to remedy some of the roller shocks. Four such wheels, however, are typically utilized to provide an omnidirectional capability to a platform (e.g., see [15]) which, since one of the wheels can temporarily lose contact with the ground on uneven terrain, may lead to significant odometry and position tracking problems.

Another interesting concept utilizing two rows of full spheres as rolling units has recently been introduced and demonstrated for omnidirectional platform motion [16]. The balls or spherical tires are arranged in two conveyor belts which produce forward and rotational motion of the platform 


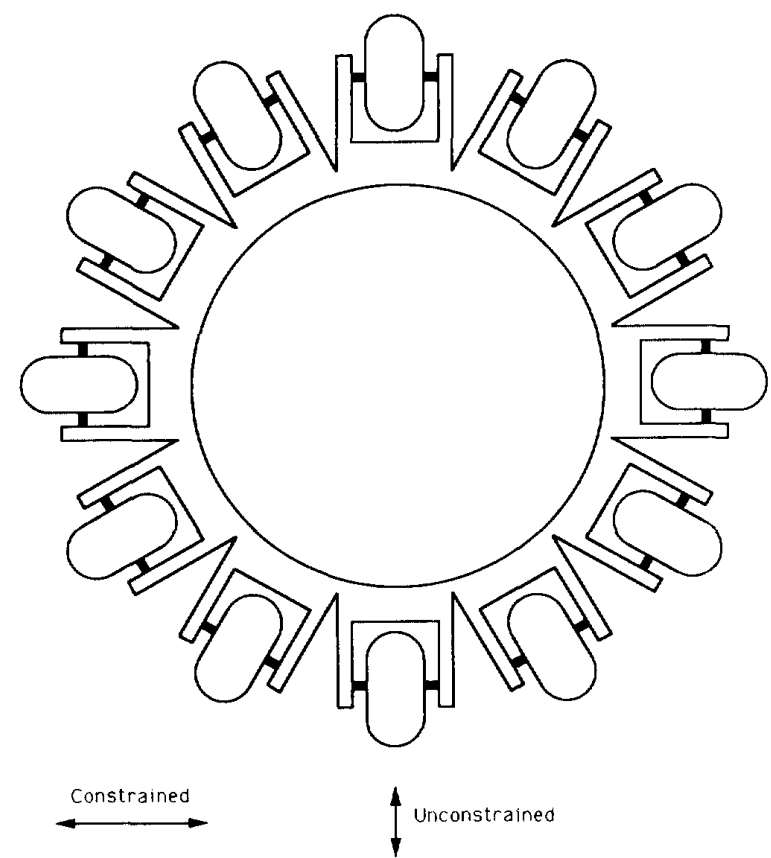

Fig. 1. Example of a "universal wheel."

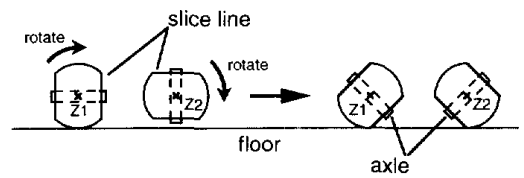

(a)
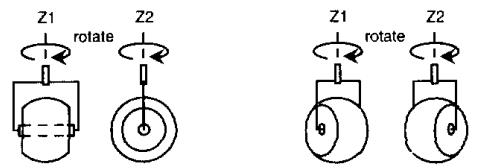

(b)

Fig. 2. Schematic of the basic orthogonal-wheels operating principle: (a) end-view, (b) top-view.

in a manner similar to that of "skid-steer" vehicles. In addition, two controlled rods in each track contact the top of the balls and, by rotating around an axis parallel to the track, provide sideways motion of the platform. Although the mechanism provides omnidirectional and holonomic motion, the rotational degree of freedom of the platform is extremely difficult to control since, like all tracked or "skid-steer" vehicles, significant sideways slippage of part of the track must occur during turns. Controlling or compensating for this tire slip on not perfectly uniform terrains does not appear feasible with the proposed design [16], and significant wear and tear of either the spherical tires or internal rods are expected on rugose ground surfaces.

In the following section, we present a novel "orthogonalwheels" concept that provides normal traction in a given direction while being free-wheeling in the other perpendicular direction. We describe the two major possible types of wheel

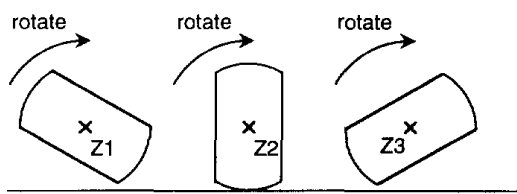

(a)

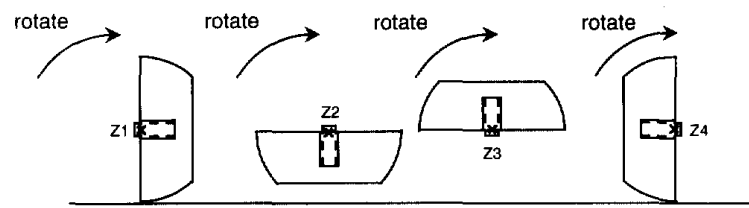

(b)

Fig. 3. Examples of alternative configurations with (a) three third-spheres and (b) four diameter-sliced spheres.

assemblies based on this concept and then show how a combination of several of these orthogonal-wheels assemblies can be used to generate an omnidirectional capability. In Section III, we apply these concepts to the design of a prototype platform that includes full omnidirectionality with independently controlled rotational and translational degrees of freedom. Some experimental results illustrating these characteristics are presented in Section IV, and concluding remarks about the applicability of the system to various robotic platforms are given in Section V.

\section{THE ORTHOGONAL-WHEELS CONCEPT AND MAJOR ASSEMBLIES}

\section{A. The Basic Principle}

The basic operating principle of the orthogonal-wheels concept is illustrated in Fig. 2. The major components are two spheres of equal diameter which have been sliced to resemble wide, rounded-tire wheels, such as those that can be found on most ATV's (All Terrain Vehicles). The axle of each wheel is perpendicular to the sliced surfaces and is mounted using ball bearings so that the wheel is freewheeling around its axle. Through a bracket which is holding the extremities of the wheel axle, each wheel can be driven to roll on its portion of spherical surface, rotating around an axis, $Z$, perpendicular to the wheel axle. When these axes ( $Z_{1}$ and $Z_{2}$, coming out of the figure plane in Fig. 2(a)) are maintained parallel and at a constant distance from each other, and when the wheel rotations around these axes are synchronized, contact with the ground can be assured by at least one wheel, while allowing enough space for the brackets holding the wheel axles to clear the ground. The end-view sketch in Fig. 2(a) shows the simplest configuration consisting of two identical wheels with $90^{\circ}$ rolling surfaces on each and with axles offset by $90^{\circ}$, rotating at the same angular velocity. Note that a variety of other configurations based on different numbers or slicing of the spheres (e.g., see Fig. 3), or various rotational speed patterns could also provide the required contact synchronization. In principle, the proper operation of the system has no requirement other than the parallelism of the $Z$ rotational axes of the wheels, constant spacing with the ground of the spheres' center, and 


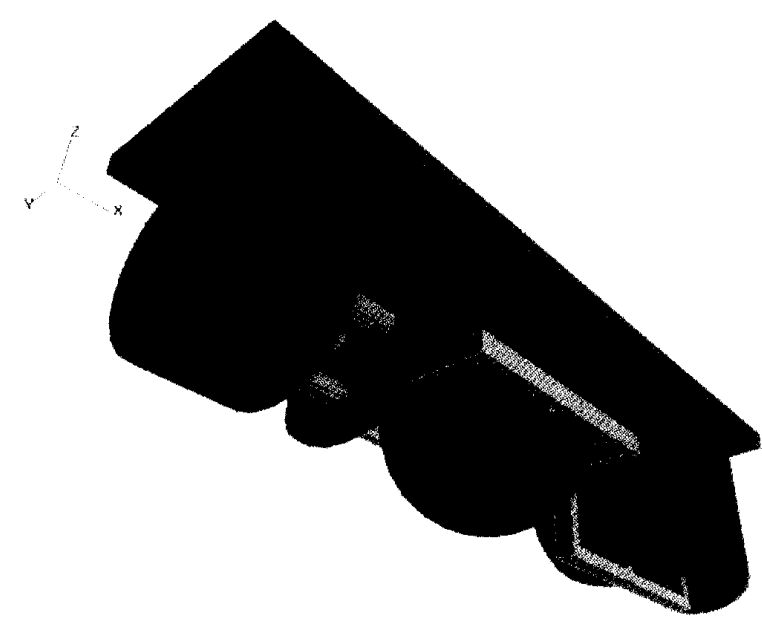

Fig. 4. Three-dimensional perspective of the "longitudinal" orthogonal-wheel assembly.

the synchronized successive contact of the wheels with the ground.

When the wheels are rotating in synchronized fashion (i.e., at the same speed for the cases shown in Figs. 2 and 3), they are driven in the direction perpendicular to the $Z$ axes. In the mean time, whatever wheel is in contact with the ground can roll freely in the direction parallel to $Z$, therefore allowing the entire wheel assembly to move freely in that direction. The two (or more) wheels do not necessarily need to be close to each other, although from a practical point of view, their proximity will minimize drive train and assembly parts. Two preferred configurations for assembling the type of orthogonal wheels shown in Fig. 2 are discussed in the following subsections.

\section{B. The Longitudinal Orthogonal-Wheel Assembly}

In this type of assembly, shown in 3-D perspective in Fig. 4, the two rotating axes (labeled $Z_{1}$ and $Z_{2}$ in Fig. 2) of the wheels are merged so that the two brackets holding the axles can be mounted at $90^{\circ}$ from each other along the axis of a common shaft. The extremities of the shaft are held in vertical plates (with ball bearings) which provide the attachment points for the assembly underneath the platform. One end of the shaft is connected to a motor that, by rotating the shaft, provides the driving of the wheel assembly.

When the main shaft turns, the wheels provide traction in the direction perpendicular to the main shaft, i.e., in the direction labeled $Y$ in Fig. 4, while they are freewheeling in the direction parallel to the shaft, i.e., direction $X$ in Fig. 4. In the direction perpendicular to the shaft, the entire assembly thus has a constrained motion which is controllable by the rotation of the main shaft, while the motion component in the direction parallel to the shaft is unconstrained. The advantages of this design over the universal wheels discussed in the previous section are: fewer needed parts, smaller wheel-well size requirements and smoother contact with the ground. Note that as long as the entire assembly is in translation, i.e., does not rotate around a vertical axis, there is no requirement for a single contact point since both wheels must move at the same velocity and have identical trajectories on the ground. This is an interesting feature for building platforms with omnidirectional translation capability only (the rotation of the upper body being provided by another independent motor above the wheel chassis as is common in platforms with three steerable wheels) since an overlap of the rolling surfaces of the two balls is feasible in order to provide a very smooth rolling behavior of the whole assembly. If rotation of this assembly around a vertical axis is desired, then a single point of contact is required to prevent slippage of one of the wheels. For the prototype platform described later in the paper, a regular machining accuracy of $.025 \mathrm{~mm}$ and the natural taper of the edges of the thin rubber films that were used on the metallic balls to improve traction provided excellent behavior during the switches of contact from one wheel to the other. As detailed in the following section, the challenge with controlling the "longitudinal" wheel arrangement shown in Fig. 4 when rotating around a vertical axis resides with how precisely the time of the contact switch can be detected, and how well the change in shaft velocity required at the switch of contact can be approximated through the control system. If the inertia of the motor and/or the wheels is so great that a good approximation is not feasible (generally resulting in a jerk in the motion and possibly in significant positioning errors), then the "lateral" wheel assembly described in the next subsection must be used to remedy this problem with some slight additional complexity in the drive train.

\section{The Lateral Orthogonal-Wheel Assembly}

The "lateral" assembly of orthogonal wheels is the most obvious from the upper part of Fig. 2, however its construction is slightly more complicated since it requires a gear- or belttype transmission. A 3-D perspective of this assembly is shown in Fig. 5. Each wheel axle is held by a bracket which is coupled to a driving shaft located on the $X$ axis of each wheel. These two driving shafts are parallel and therefore can be coupled by gears or, as shown in Fig. 5, by a transmission belt so that they always turn at the same velocity, driven by a common motor. The assembly thus has a constrained and controllable motion in the direction perpendicular to the shafts and labeled $Y$ in Fig. 5, while it is freewheeling in the direction parallel to the shafts and labeled $X$ in Fig. 5.

Use of this "lateral" arrangement of the wheels has been proposed for some mechanisms with bi-directional motion capabilities [17]; however, as will be shown here, not only can full three-DOF omnidirectionality be achieved with proper control of the constrained and unconstrained directions of motion, but also rotation of the assembly around any vertical axis takes place with no slippage of the wheels and without discontinuity in the motor speed: consider Fig. 6 in which the centers of the two spheres enveloping the wheels of a lateral assembly have been denoted by points $L$ and $R$. The constrained and freewheeling directions of motion of the assembly are denoted by the vectors $\vec{C}$ and $\vec{F}$, respectively. Assume that the entire assembly is performing an arbitrary motion in the $(x, y)$ plane, and let $L(t)=\left(x_{L}(t), y_{L}(t)\right)$ and $R(t)=\left(x_{R}(t), y_{R}(t)\right)$ denote the paths of the points $L$ and 


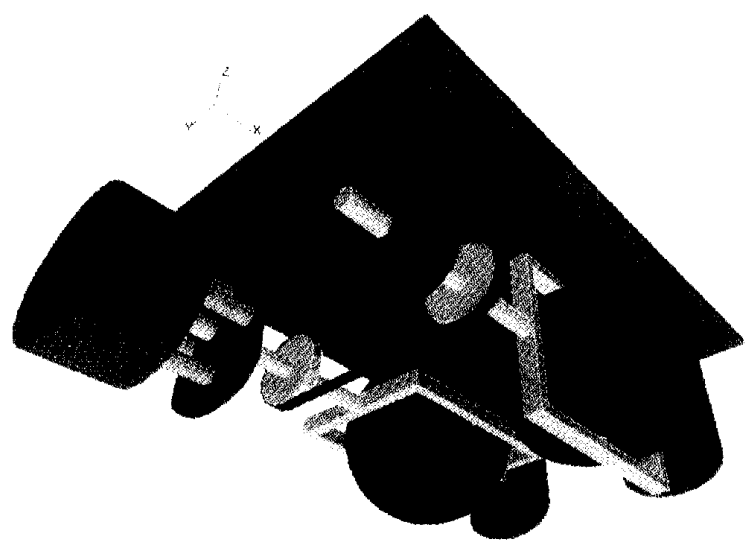

Fig. 5. Three-dimensional perspective of the "lateral" orthogonal-wheel assembly.

$R$. Since the assembly's body is rigid, the distance between $R$ and $L$ (i.e., the norm of the vector $\overrightarrow{R L}=L(t)-R(t)$ must remain constant:

$$
\frac{d}{d t}((L(t)-R(t)) \cdot(L(t)-R(t)))=0 .
$$

Denoting by $\vec{V}_{L}$ and $\vec{V}_{R}$ the respective velocities of $L$ and $R$, (1) gives:

$$
\vec{V}_{L} \cdot(L(t)-R(t))=\vec{V}_{R} \cdot(L(t)-R(t))
$$

or

$$
\vec{V}_{L} \cdot \overrightarrow{R L}=\vec{V}_{R} \cdot \overrightarrow{R L}
$$

Thus, the rigid body constraint requires that either the velocity vectors of the two points be equal (in which case the body performs translational motion), or that the projections of the velocity vectors on the line joining the two points be equal. ${ }^{2}$ For the lateral assembly in which the constrained direction of the wheels is along the line joining the two wheels, (3) implies that, during any motion of the assembly, both wheels are required to turn at the same speed in their constrained direction. Therefore, no discontinuous change in the motor speed is necessary when ground contact switches from one wheel to the other. Moreover, both wheels could be in contact with the ground (i.e., an overlap in the rolling surfaces of the two wheels is allowed), and no slippage would occur since the motor drives both wheels at the same speed in the constrained direction. Fig. 6 schematically illustrates this property for a lateral assembly rotating around an instantaneous center of

\footnotetext{
${ }^{2}$ Since many rolling assemblies, other than the two presented here, could be envisioned and/or designed based on the concepts shown in Figs. 2 and 3 , or on other similar concepts, the importance and generality of (3) is worth emphasizing here. Since it represents a consequence of the general rigid body constraint between two points. (3) can be applied to the center points of any pair of wheels in an assembly, with the implication for any single-motor driven assembly that, if the constrained direction of motion is not parallel to the line joining the wheel centers, then some slippage will occur when both wheels make contact with the ground (during the switch of contact, or if an overlap of the rolling surfaces exists) during any non-translational motion of the assembly.
}

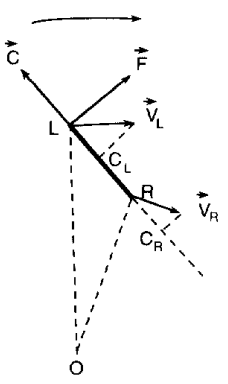

Fig. 6. Schematic of a lateral assembly rotating around a vertical axis.

rotation, denoted by point $O$. In the notation of the figure, (3) translates into

$$
C_{L}=C_{R}
$$

where $C_{L}$ and $C_{R}$ represent the projections of the velocity vectors $\vec{V}_{L}$ and $\vec{V}_{R}$ on the constrained $\vec{C}$ direction, i.e., on the line joining the two points $L$ and $R$ in this case. Note that the projections of $\vec{V}_{L}$ and $\vec{V}_{R}$ on the $\vec{F}$ direction are not equal, but this is inconsequential for slip analysis since the wheels are freewheeling in that direction.

\section{Generating Omnidirectionality with Orthogonal-Wheel Assemblies}

Both lateral and longitudinal wheel assemblies can be used in the same manner to provide an omnidirectional capability to platforms: when placing two or more of these assemblies underneath a platform, their respective motion in constrained directions can be combined to produce a motion of the platform in any desired direction, while each assembly freewheels in its unconstrained direction. For example, consider the arrangement shown schematically in Fig. 7 where two assemblies are attached under a chassis. The constrained and unconstrained directions of each assembly are denoted by the letters $c$ and $u$, respectively. If the platform needs to move in the $x$ direction with the linear velocity $V_{x}$ (in $\mathrm{m} / \mathrm{sec}$ ) then the motor of assembly 1 needs to turn clockwise at a velocity (in revolution per second) $w=V_{x} / 2 \pi R, R$ being the radius (in $\mathrm{m}$ ) of the wheels' sliced spheres, while the motor of assembly 2 is not turning so that assembly 2 is only freewheeling during the motion. If the platform needed to move at a velocity $V$ oriented at $45^{\circ}$ from the $x$ direction, then the motor of assembly 1 would need to turn at the velocity $w=(V / 2 \pi R) / \sqrt{2}$ (clockwise) and the motor of assembly 2 would need to turn at the velocity $-w$ (counterclockwise), while both assemblies would be freewheeling at velocity $V / \sqrt{2}$ in their respective unconstrained directions. Thus, by appropriately positioning several assemblies under a platform, and by properly combining their constrained directions of motion, it is possible to drive and control a platform in any direction. As shown in the next section, the system can also be given a rotational capability which, for certain configurations of the assemblies, can be controlled independently of the translational capability. 


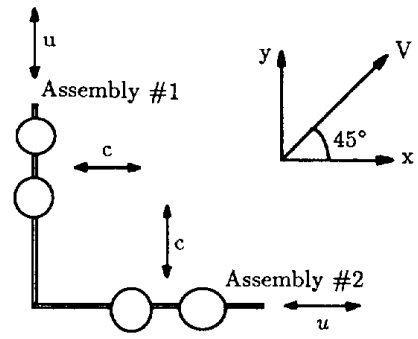

Fig. 7. Schematic of the combination of constrained and unconstrained directions of motion for two orthogonal-wheels assemblies.

\section{DESIGN OF OMNIDIRECTIONAL AND HOLONOMIC PLATFORMS}

\section{A. Basic Layout With Three Assemblies}

Many options are available for positioning the wheel assemblies in the design of an omnidirectional mobile platform. The only requirements are that the layout provide enough directions of constrained motions of the assemblies to allow both omnidirectional translation and rotation of the platform, and that stability of the platform be maintained independently of the internal configuration of the assemblies, i.e., which wheel in each assembly makes ground contact. To produce a platform with three full degrees of freedom, the simplest layout with no kinematic redundancy requires three assemblies of the type shown in Figs. 4 or 5. With the three assemblies located at the three apexes of a triangle, the platform load stability is extremely easy to ensure, while a $120^{\circ}$ orientation relationship between the three constrained motion directions provides excellent directional control capability. The schematics of two such layouts using longitudinal and lateral wheel assemblies are shown in Fig. 8(a) and (b), respectively. Note that, without the benefit of a suspension system, a layout with four perpendicular assemblies would not provide added load carrying capability and, in some cases, would invalidate the directional control because of the possibility for non-contact of one of the wheels on uneven ground.

\section{B. Kinematic Relationships}

In both schematics of Fig. 8, the constrained directions of motion of each assembly are indicated by the arrows labeled 1,2 , and 3 . For each layout, let $\dot{\psi}$ denote the angular velocity (in $\mathrm{rad} / \mathrm{sec}$ ) of the internal reference frame of the platform ( $X_{\text {ref }}, Y_{\text {ref }}$ ) with respect to an absolute reference frame $(x, y)$. The magnitude of the platform translational velocity (in $\mathrm{m} / \mathrm{s}$ ) is denoted by $|V|$ and its direction with respect to the platform internal reference frame is denoted by $\Theta \in[0,2 \pi]$. With these conventions, the wheels' driving shaft velocities, $w_{i}$, can be calculated (in $\mathrm{rad} / \mathrm{sec}$ ) for either layout in Fig. 8 as:

$$
\begin{aligned}
& w_{1}=\frac{|V|}{2 R}(\sin \Theta-\sqrt{3} \cos \Theta)+\frac{\dot{\psi} L_{1}}{R} \\
& w_{2}=-\frac{|V|}{R} \sin \Theta+\frac{\dot{\psi} L_{2}}{R} \\
& w_{3}=\frac{|V|}{2 R}(\sin \Theta+\sqrt{3} \cos \Theta)+\frac{\dot{\psi} L_{3}}{R}
\end{aligned}
$$

where $R$ is the radius of the spherical wheels and $L_{i}$ represents the distance between the center of the platform and the center of the wheel of assembly $i$ currently contacting the ground. The first terms on the right-hand side of (5)-(7) represent the projections of the translational velocity $|V|$ on the constrained motion directions of each assembly, while the last terms represent the components due to the rotational velocity of the platform.

As discussed in the following section, (5)-(7) represent a very convenient formulation of the platform kinematics for the sensor-based and trajectory-tracking control modes. In teleoperation and for odometry calculations, a slightly modified form of these equations can be used, which clearly illustrates the one-to-one relationship which exists between the Cartesian and joint velocities: if we set

$$
\begin{aligned}
& V_{x}=|V| \cos \Theta \\
& V_{y}=|V| \sin \Theta
\end{aligned}
$$

Equations (5)-(7) can be written as:

$$
\left(w_{1}, w_{2}, w_{3}\right)^{T}=A\left(V_{x}, V_{y}, \dot{\psi}\right)^{T}
$$

with

$$
A=\frac{1}{R}\left(\begin{array}{ccc}
-\frac{\sqrt{3}}{2} & \frac{1}{2} & L_{1} \\
0 & -1 & L_{2} \\
\frac{\sqrt{3}}{2} & \frac{1}{2} & L_{3}
\end{array}\right)
$$

Since $L_{1}, L_{2}$, and $L_{3}$ are always positive quantities, $A$ is invertible and its inverse matrix $A^{-1}$ is:

$$
A^{-1}=\frac{R}{L_{1}+L_{2}+L_{3}}\left(\begin{array}{ccc}
\frac{-2 L_{3}-L_{2}}{\sqrt{3}} & \frac{L_{1}-L_{3}}{\sqrt{3}} & \frac{2 L_{1}+L_{2}}{\sqrt{3}} \\
L_{2} & -L_{1}-L_{3} & L_{2} \\
1 & 1 & 1
\end{array}\right)
$$

so that

$$
\left(V_{x}, V_{y}, \dot{\psi}\right)^{T}=A^{-1}\left(w_{1}, w_{2}, w_{3}\right)^{T}
$$

It is clear from these relations that the rotational and translational motions are fully decoupled and can be controlled independently and simultaneously. Moreover, there are no constraints on the space of achievable translational and rotational velocities of the platform, and (5)-(7) or (10)-(13) represent a bijective mapping between the sets of $\left(w_{1}, w_{2}\right.$, $\left.w_{3}\right)$ and $(\Theta,|V|, \dot{\psi})$ or $\left(w_{1}, w_{2}, w_{3}\right)$ and $\left(V_{x}, V_{y}, \dot{\psi}\right)$. In their three-dimensional space of planar motion (translation and rotation), both platforms illustrated in Fig. 8(a) and (b) are thus omnidirectional and holonomic systems. Moreover, both platforms can fully access the three degrees of freedom of planar rigid body motions with no constraints or compatibility conditions on the three independent controls, a capability not achieved by any conventional wheeled system.

Although (5)-(13) apply to either layout in Fig. 8, the effect of the different characteristics of the assemblies with respect 

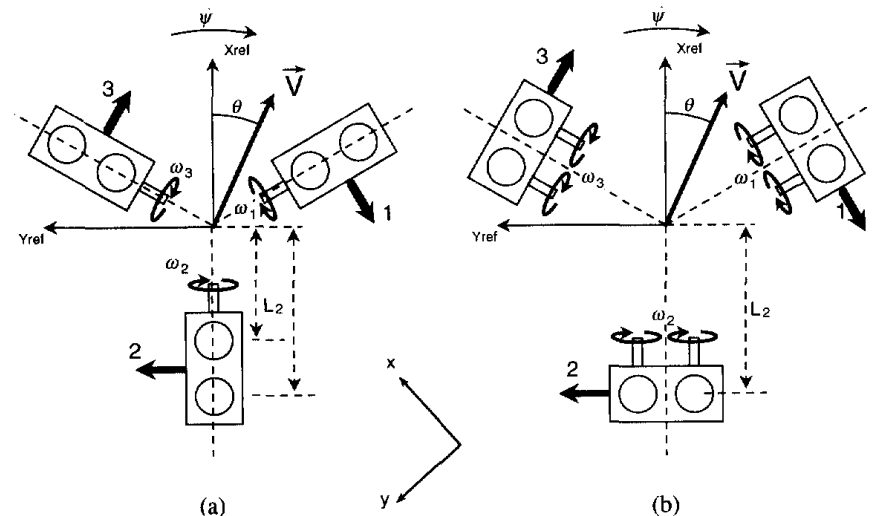

(b)

Fig. 8. Schematic of the basic layout for three-DOF motion using (a) longitudinal and (b) lateral orthogonal-wheel assemblies.

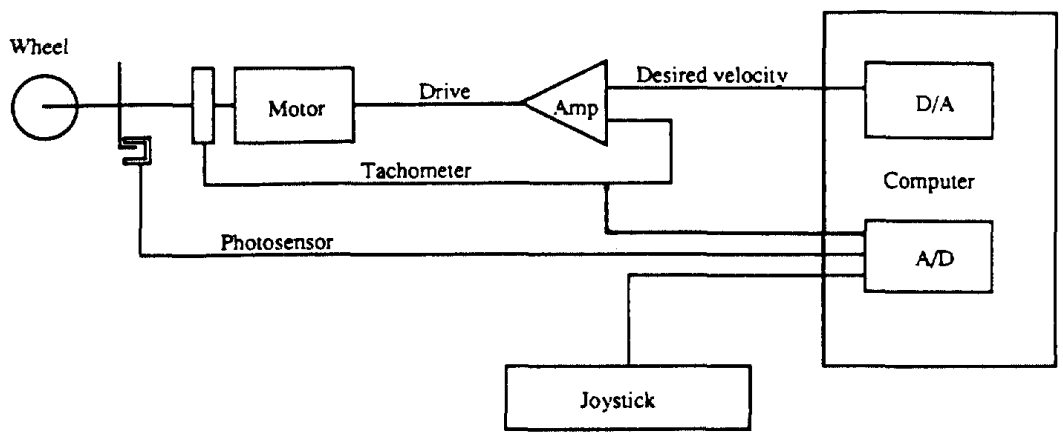

Fig. 9. Control block diagram for the orthogonal wheel assemblies.

to rotation around a vertical axis are quite explicit here: for the case of Fig. 8(b) using the lateral assemblies, the values of $L_{1}, L_{2}$, and $L_{3}$ are the same whichever wheel in the corresponding assembly is in contact with the ground, whereas in the case of Fig. 8(a), these values change when contact with the ground switches from one wheel to the other in the longitudinal assemblies. This is illustrated in Fig. 8(a) by the two dimension arrows showing the two possible values of $L_{2}$. From the control point of view, rotational motion of the longitudinal assembly thus represents a challenge since the switch of contact from one wheel to the other and the corresponding step function in $w_{i}$ have to be accurately tracked and implemented. As far as translational motions are concerned, the two layouts are kinematically equivalent (with the same constant coefficients in the first two columns of the matrix $A$ ) and, to produce platforms with omnidirectional translational capabilities only, the layout of Fig. 8(a) using the much-simpler-to-fabricate longitudinal assembly would probably be preferred. For full three-DOF platforms needing a rotational capability, the layout of Fig. 8(b) using the lateral wheel assemblies should be selected because of the constant values of the $L_{i}$ 's and the much simpler control system.

\section{Control System}

To verify the proposed concepts, an experimental platform was constructed with longitudinal wheel assemblies and an on-board computer-based control system. The computing hardware is composed of a VME bus with seven slots occupied by a $68020 \mathrm{CPU}$, a floppy controller, a hard disk controller, serial ports, D/A and A/D cards and a custom-designed VLSI fuzzy logic inferencing board [18]-[20]. Each orthogonalwheel assembly is driven by a typical variable speed de motor equipped with a tachometer to provide feedback to the velocity control. Photosensors were also added on the main shaft of the longitudinal wheel assemblies to track ground contact of the wheels.

Fig. 9 shows the basic control block diagram for one of the assemblies. The amplifier is set up in a velocity loop with feedback from the tachometer. Data from the three tachometers are also fed back to the computer to perform the dead reckoning. The commands to the velocity control loops are provided at $100 \mathrm{~Hz}$ by the computer which receives input either from a joystick for operation in teleoperated mode, or from the path planning and tracking modules in autonomous mode.

In teleoperated mode, the signals from the joystick directly provide the values of $\dot{\psi}, V_{x}$, and $V_{y}$. The control system calculates the three shaft velocities from (10) and (11) and servos on these at $100 \mathrm{~Hz}$ using the tachometer data. In autonomous mode, the input to the control system are either "target configurations" $(x, y, \psi)$ which are provided by the user in a list of "via points" forming a trajectory, or "target speeds" $(|V|, \Theta, \dot{\psi})$ calculated by the reasoning systems 


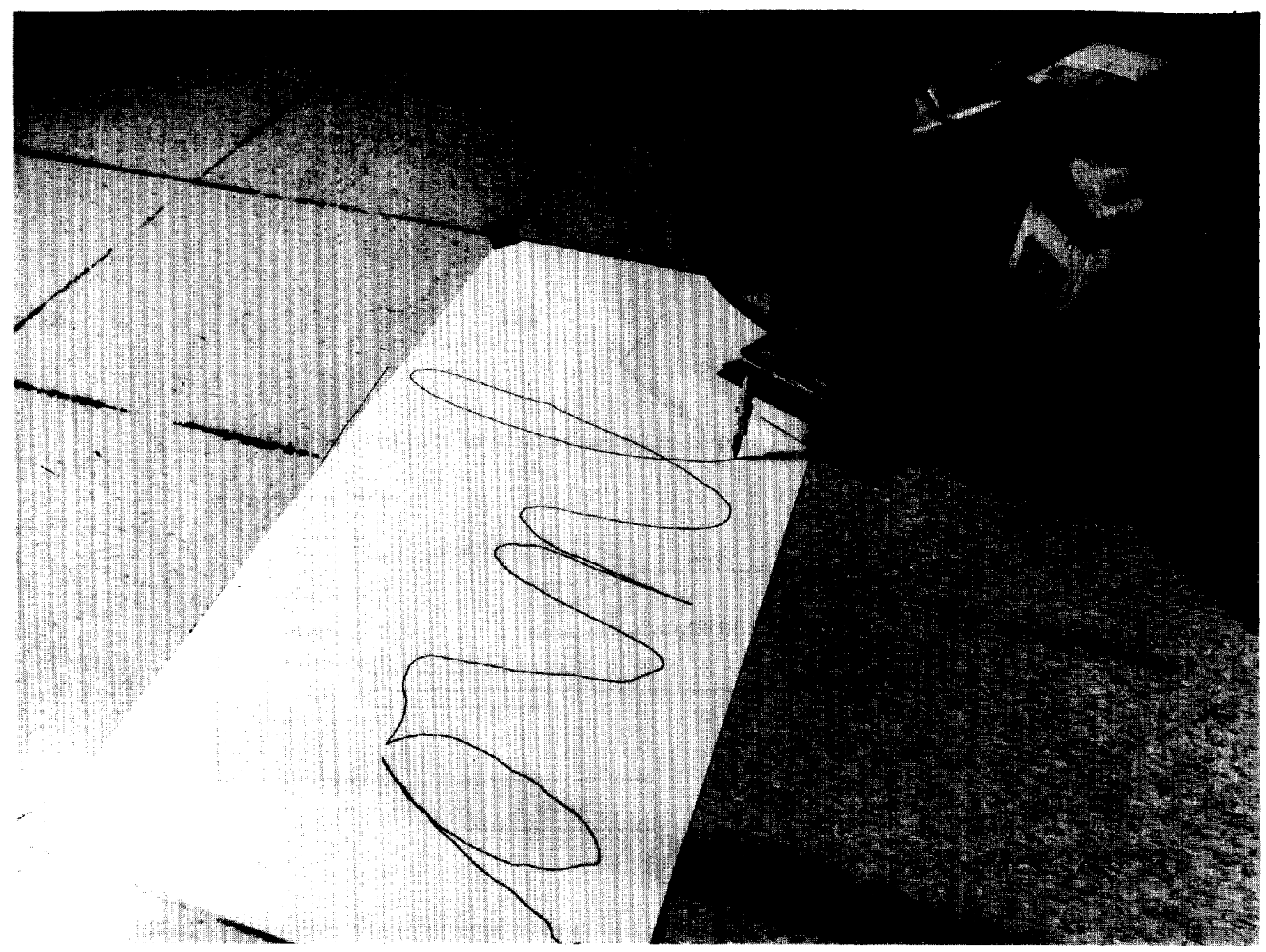

Fig. 10. Platform performing a trajectory requiring translational omnidirectionality.

at sensor sampling rate during sensor-based navigation. The inferencing modules for obstacle avoidance and decisionmaking in sensor-based navigation, which use a ring of twentyfour acoustic range sensors on the deck of the platform and the custom-designed VLSI fuzzy logic inferencing board, are described in detail in [21], [22]. In the user-provided trajectory following mode, the target configuration is compared at every loop cycle to the current estimate of position and orientation calculated by the dead reckoning. The results provide the desired direction of motion and the platform target rotational and translational speeds using linear ramp up profiles, up to the preset maximum velocities. The corresponding shaft velocities, $w_{\text {iest }}$, are calculated from (5)-(7) and are used to check that the maximum allowed shaft velocity is not exceeded. If this is the case, all velocities (the system in (5)-(7) is linear with respect to $|V|$ and $\dot{\psi}$ ) are decreased by the ratio $w_{\text {iest }} / w_{\text {imax }}$ prior to being fed to the servo controls. When the platform comes within a radius $r_{\text {slow }}$ from its target location or within an angle, $\psi_{\text {slow }}$, from its target orientation, the calculated translational velocity, $|V|$, or the rotational velocity, $\psi$, are decreased using linear ramp down profiles. When the location and rotation angle are both within given thresholds, $r_{\text {new }}$ and $\psi_{\text {new }}$, from their target values, a new entry is read from the list and becomes the target configuration, or the platform stops if the list is exhausted.

At each loop cycle (of length $\Delta t$ ), the dead reckoning system integrates the rotational and translational velocities to estimate the current orientation and position of the platform. With the values of the shaft velocities, $w_{i}^{*}$, fed back during the loop cycle and the distance, $L_{i}$, obtained from the photosensors data, the platform velocities are easily calculated from (12) and (13). The cycle rotational increment, $\Delta \psi$, is first calculated from the rotational speed, and the translational velocities are integrated to yield the displacement increments in the platform reference frame. The $\Delta x$ and $\Delta y$ increments in the absolute reference frame are then calculated using the linearly estimated mid-loop value of $\psi$

\section{EXPERIMENTAL RESULTS}

To demonstrate the operationality of the orthogonal-wheels assembly concept and to test the control scheme and dead reckoning systems described in the previous section, a series of tests was performed in which a variety of trajectories, each specified as a list of "via points," were submitted to the testbed platform control system. Fig. 10 shows the platform during 


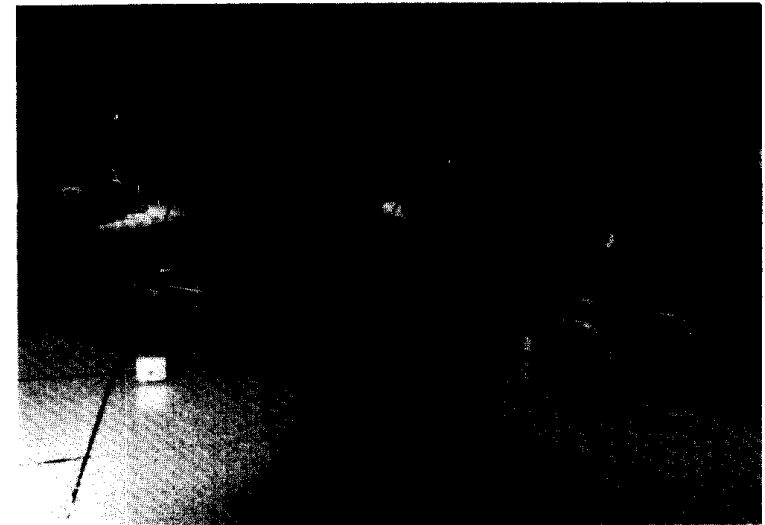

Fig. 11. Platform performing a path tracking test trajectory.

one of these tests made to illustrate its translational omnidirectionality. A pen attached to the side of the platform is used to display the trajectory consisting of points, approximately $3 \mathrm{~cm}$ apart, that were digitized from an actual hand-written note. The ability of the platform to translate in any direction is quite apparent, while that of rapidly changing direction of motion is demonstrated, for example, at the top of the letter " $o$ " or the bottom of the letter " $n$." The trajectory was closed by asking the platform to "frame" the writing and return to its starting location. The error shown by the position of the pen when the platform stopped was about $2 \mathrm{~cm}$ for this trajectory which was about $6 \mathrm{~m}$ in length. Longer trajectories such as those shown in Fig. 11 were also used to investigate the accuracy of the dead reckoning system and the platform's path tracking capabilities.

The photograph of Fig. 11 was taken as a time exposure in a dark room. The actual trajectory of the platform is depicted by the light trace of the small computer LED's. At discrete times during the experiment, a flash was burned to illuminate the platform and show some of its positions while moving on the trajectory. In repeated experiments with this and other trajectories, the errors during translational motions were found always much less than $1 \%$ of the length of the trajectory. More importantly, analysis of the end point errors in repeated trajectories showed that a large component $(80 \%)$ of the position error is systematic, and therefore is further reducible through an improved dead reckoning system. For this reason, our next experimental platform is planned to incorporate encoders on the shafts of the assemblies to minimize the errors due to velocity integration schemes.

Fig. 12 shows a sequence of pictures which illustrate the platform capability for simultaneous motions in translation and rotation. A small triangular flag has been added on the platform to display its "spinning" motion while it translates along a straight line. As indicated by (10)-(13) the control of the rotational and translational velocities of the platform are fully decoupled and independent, and this was verified through a series of experiments involving various speed patterns and variable rotational velocities. As expected while using the longitudinal type of assembly, the errors for the rotational component of the motions were found worse than those for the translational motions, with errors reaching $3 \%$ of the total rotation in some trajectories with fast (near the motor limits) rotational motions. As discussed previously, detection of the exact time at which ground contact switches from one ball to the other in the longitudinal assembly is critical, and a good approximation of the step functions must be feasible with the motors. For the small inertia platform dealt with here, the change in motor speed was accomplished in a single loop cycle time; however, even with the $100 \mathrm{~Hz}$ loop rate utilized here, the $\pm .01 \mathrm{~s}$ approximation of the switch times led to random errors which progressively accumulated in some trajectories. The "lateral" orthogonal-wheels assembly does not suffer from these errors and therefore, should serve as the basic assembly for platforms that require a rotational motion component.

\section{CONCLUding REMARKS}

An original orthogonal-wheels assembly concept which exhibits constrained and unconstrained directions of motion has been presented. Two possible assembly configurations of the wheels, labeled the "longitudinal" and "lateral" assemblies, have been discussed and their use in producing omnidirectionality of platforms through various combinations of their constrained motions has been described. A design has been proposed to produce fully omnidirectional and holonomic platforms with unconstrained, simultaneous, and independently controlled rotational and translational motions. A prototype of such a platform has been constructed and its control system has been described. Proof-of-principle experiments illustrating the orthogonal-wheels assembly concept and the platform omnidirectionality with simultaneous and independently controlled translational and rotational motions have been presented. Data from the experiments with this first prototype suggest that very accurate control of the omnidirectional translation motions can be obtained using either the "lateral" or "longitudinal" orthogonal-wheels assemblies, and that significantly better control of the platform rotational motions can be realized using the "lateral" type of assemblies at the cost of a slight additional complexity in the design of the drive trains.

Besides their overall holonomy and omnidirectionality, the very important and unique characteristic of mobile platforms based on orthogonal-wheels assemblies is that the controls of their rotational and translational motions are fully decoupled. In other words, the platforms have three independent controls that can be used to fully access the three degrees of freedom of planar rigid body motions with no constraints or compatibility conditions on the controls. This feature, and the associated one-to-one relationship between Cartesian and joint velocities, is particularly interesting with respect to accurate path tracking and positioning since loop-rate errors in the actuation controls would result in platform positioning errors that are directly tractable with encoders, rather than in wheel slippage as is the case when steering and velocity compatibility conditions are even slightly violated in conventional wheel systems. Our ongoing work focuses on experimental studies in this area using a second prototype platform equipped with "lateral" 


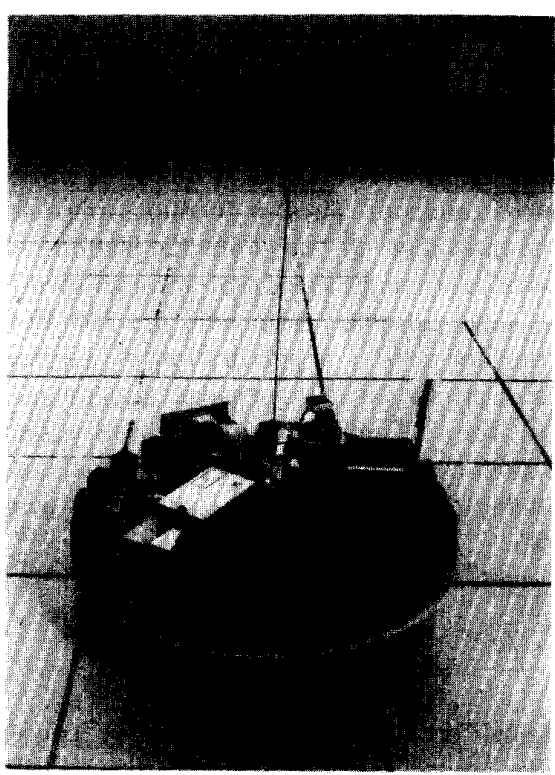

(a)

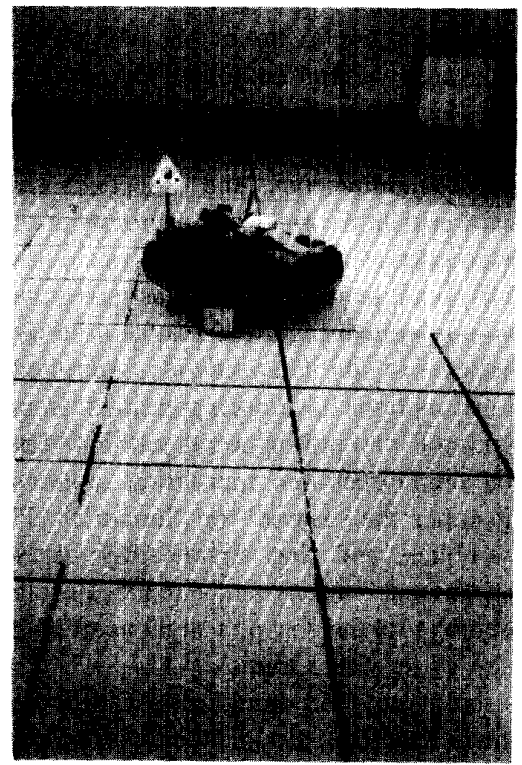

(c)

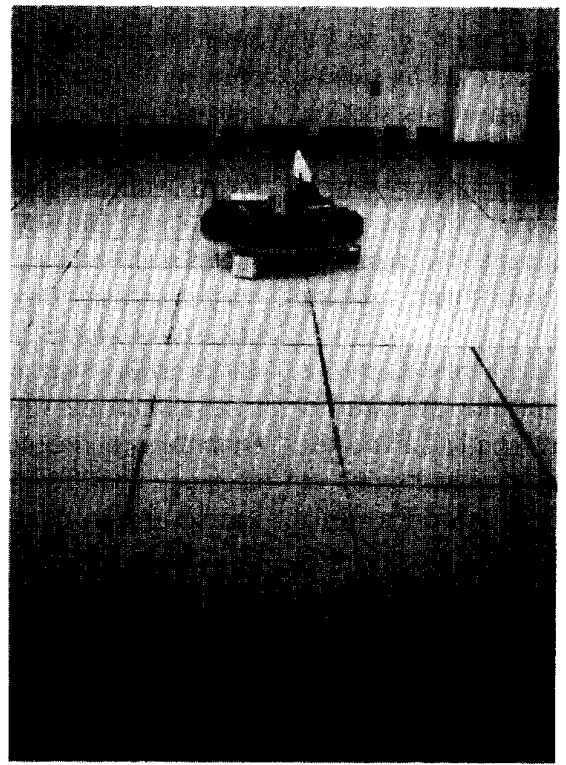

(d)

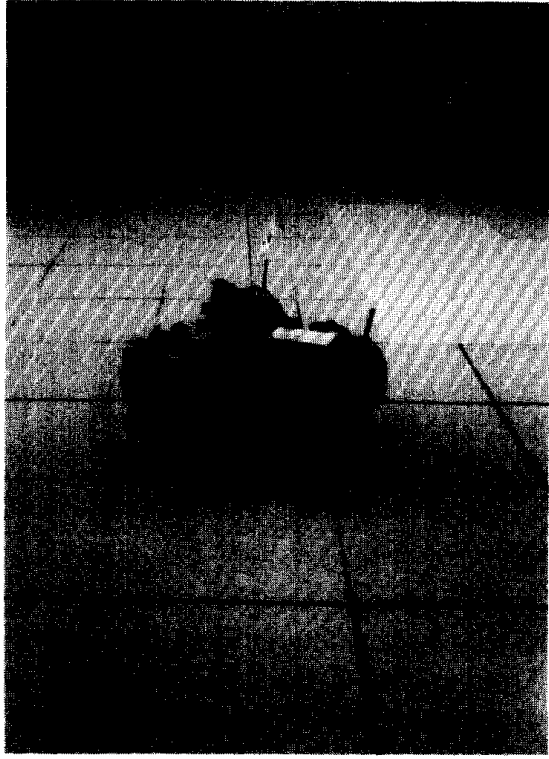

(b)

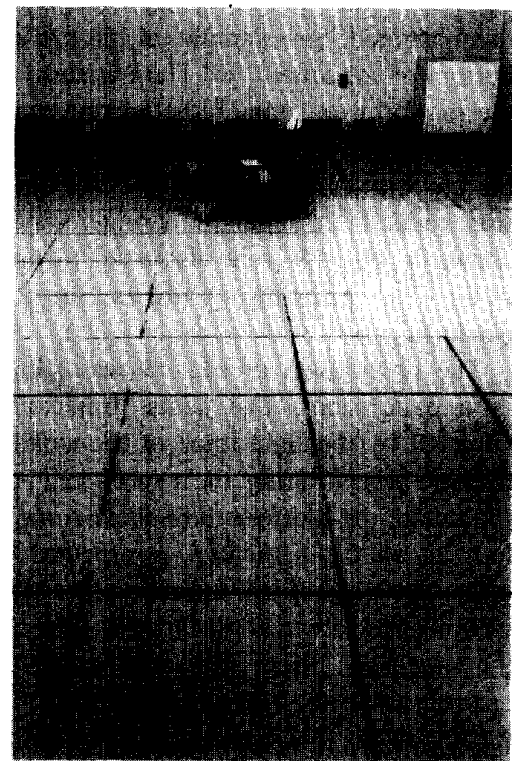

(e)

Fig. 12. Sequence of pictures illustrating the simultaneous rotation and translation capability of the platform.

orthogonal-wheel assemblies and encoders on the shaft of the assemblies.

The basic platforms discussed in this paper can thus be viewed as omnidirectional, holonomic, statically stable, and fully controllable "casters." The fact that their omnidirectionality is achieved without the steering of wheels around a vertical axis (and the associated friction and possible tear) is particularly interesting for the design of large, possibly oddshaped robotic platforms or vehicles having to operate and maneuver in constricted spaces. Furthermore, proper coordinated control of several of these low profile (small wheel well) casters underneath a large platform would in turn provide full omnidirectionality to that platform.

Another envisionable general area of applications would involve positioning the proposed rolling platforms "wheels up" in transporting or conveyor-type systems so that large packages, cargo-handling pallets, parts to be machined, etc., can be routed or accurately positioned. The omnidirectionality, decoupled controls, and no-slippage motion capabilities would allow to precisely and simultaneously translate and rotate the transported materials without leaving undesirable scratches or friction marks. 


\section{REFERENCES}

[1] P. F. Muir and C. P. Neuman, "Kinematic modeling of wheeled mobile robots," J. Robotic Syst., vol. 4, pp. 281-340, 1987.

[2] J. P. Laumond, "Feasible trajectories for mobile robots with kinematic and environment constraints," in Proc. Intell. Autonomous Syst. Conf., Amsterdam, The Netherlands, Dec. 1986.

[3] F. G. Pin and H. A. Vasseur, "Trajectory generation for autonomous mobile robots with non-holonomic and steering angle constraints," in Proc. IEEE Int. Workshop Intell. Motion Control, Istanbul, Turkey, Aug. 1990 , pp. $295-299$

[4] H. A. Vasseur, F. G. Pin, and J. R. Taylor, "Navigation of a car-like mobile robot in obstructed environments using convex polygonal cells," Robotics and Autonomous Syst., vol. 10, no. 2-3, pp. 133-146, 1992.

[5] G. Giralt, R. Chatila, and M. Vaisset, "An integrated navigation and motion control system for autonomous multisensory mobile robots," in Robotics Res.: First Int. Symp. Cambridge, MA: MIT Press, 1984.

[6] Y. Kanayama and B. I. Hartman, "Smooth local path planning for autonomous vehicles," in Proc. IEEE Int. Conf. Robotics Autom., Scottsdale, AZ, May 1989, pp. 1265-1270.

[7] C. R. Weisbin, G. de Saussure, J. R. Einstein, F. G. Pin, and E. Heer, "Autonomous mobile robot navigation and learning," Computer, vol. 22 , no. 6, pp. 29-35, 1989.

[8] F. G. Pin, M. Beckerman, P. F. Spelt, J. T. Robinson, and C. R. Weisbin, "Autonomous mobile robot research using the HERMIES-III robot," in Proc. IROS Int. Workshop Intell. Robot and Syst., Tsukuba, Japan, Sept. 1989, pp. 251-256.

[9] D. B. Reister, "A new wheel control system for the omnidirectional HERMIES-III robot,"Robotica, vol. 10, pp. 351-360, 1992.

[10] R. A. Brooks, "Elephants don't play chess," Robotics and Autonomous Syst., vol. 6, no. 1-2, pp. 3-15, 1990 .

[11] R. C. Arkin, "Integrating behavioral, perceptual, and world knowledge in reactive navigation," Robotics and Autonomous Syst., vol. 6, no. 1-2. pp. $105-122,1990$.

[12] Y. Koren and J. Borenstein, "Potential field methods and their inherent limitations for mobile robot navigation," in Proc. IEEE Int. Conf Robotics Autom., Sacramento, CA, Apr. 1991, pp. 1398-1404.

[13] J. C. Alexander and J. H. Maddocks, "On the kinematics of wheeled mobile robots," Int. J. Robotics Res., vol. 8, no. 5, pp. 15-27, 1989.

[14] D. B. Reister and M. A. Unseren, "Position and constraint force control of a vehicle with two or more steerable drive wheels," IEEE Trans Robotics Automat.. vol. 9, no. 6, pp. 723-731, 1993.

[15] G. L. Blaisdell, "Performance of an omnidirectional wheel on snow and ice," Naval Eng. J., vol. 103, no. 1, pp. 34-41, 1991

[16] M. West and H. Asada, "Design of a holonomic omnidirectional vehicle" in Proc. IEEE Int. Conf. Robotics Autom., Nice, France, May 1992, pp. 97-103.

[17] W. Rose, "Powered spheres 'walk' and roll," Eureka, pp. 70-72, Oct. 1988.

[18] F. G. Pin, H. Watanabe, J. Symon, and R. S. Pattay, "Using custom designed VLSI fuzzy inferencing chips for the autonomous navigation of a mobile robot," in Proc. IROS'92, IEEEIRS,I Int. Conf. Intell. Robots and Syst., Raleigh, NC, July 1992, pp. 790-795.

[19] H. Watanabe, W. Dettloff, and E. Yount, "A VLSI fuzzy logic inference engine for real-time process control," IEEE J. Solid-State Circ., vol. 25 pp. 376-382, 1990.
[20] J. R. Symon and H. Watanabe, "Single board system for fuzzy inference," in Proc. Workshop Software Tools for Distributed Intell. Control Syst., Sept. 1990, pp. 253-261.

[21] F G. Pin, H. Watanabe, J. R. Symon, and R S. Pattay, "Autonomous navigation of a mobile robot using custom-designed qualitative reasoning VLSI chips and boards," in Proc. IEEE Int. Conf. Robotics Automat., Nice, France, May 1992, pp. 123-128.

[22] F. G. Pin and Y. Watanabe "Steps toward sensor-based vehicle navigation in outdoor environments using a fuzzy behaviorist approach." Int J. Intell. and Fuzzy Syst., vol. 1, no. 2, pp. 95-107, 1993.

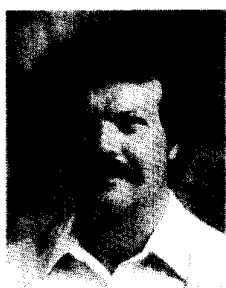

Françcois G. Pin (M'92) received the Maitrise de Mécanique from the Université de Nancy 1, France, and the Diplome National d'Ingénieur Electromécanicien from the Ecole Nationale Supérieure d'Electricité et Mécanique de Nancy, France. He received the M.S. and Ph.D. in mechanical engineering and aerospace sciences from the University of Rochester, NY.

$\mathrm{He}$ is the Group Leader of the Autonomous Robotic Systems Group at the Oak Ridge National Laboratory, Oak Ridge, TN. He is also a principal investigator of the Center for Engineering Systems Advanced Research (CESAR) Program. He joined the research staff of the Oak Ridge National Laboratory in 1982, at which time he conducted the major part of his research work in the area of mathematical and numerical modeling. His current research work and interests include the development of methodologies for the real-time control of intelligent machines, and, in particular, for the sensorbased operation in unstructured environments of mobile robots, combined mobility/manipulation systems, and man-machine synergistic systems. He has authored or co-authored more than 100 publications.

Dr. Pin serves on the program committee of many intelligent systems and robotics-related conferences, and is on the editorial boards of several international journals.

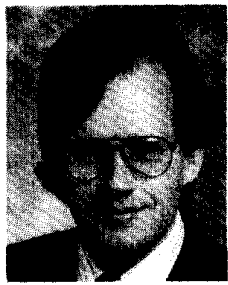

Stephen M. Killough (M'86) received the B.S.E.E. degree from the Georgia Institute of Technology in 1977 and the M.S.E.E. degree from the University of Tennessee in 1983

Since joining the Oak Ridge National Laboratory, Oak Ridge, TN, in 1983, he has been a member of the Robotics and Process Systems Division there. $\mathrm{He}$ is presently involved in robotics for remote maintenance of nuclear facilities and remote radioactive waste clean-up operations. 